\title{
Do Faster Food and Drug Administration Drug Reviews Adversely Affect Patient Safety? An Analysis of the 1992 Prescription Drug User Fee Act
}

\author{
Henry Grabowski Duke University \\ Y. Richard Wang Temple University Hospital
}

\begin{abstract}
Food and Drug Administration (FDA) review times have significantly declined under the user-fee regime. This situation has provoked concerns that drug safety has been adversely affected. Combining information from several comprehensive databases, we analyze how the FDA's review time, a drug's novelty, and a lag between the foreign and U.S. launches of a drug affect the number of serious adverse events associated with new-drug introductions in the United States in 1992-2002. We find that more novel drugs, those with shorter U.S. launch lags, and those with black-box warnings have a larger number of serious adverse events. After controlling for these and other factors, we find no association between the FDA's review time and adverse events. Because many serious adverse events involve rare occurrences that are not observable in premarket clinical trials, policy makers should direct increased agency attention and resources to postmarketing surveillance.
\end{abstract}

\section{Introduction}

Do faster new-drug reviews adversely affect patient safety? This has become an important regulatory policy issue. The 1992 Prescription Drug User Fee Act (PDUFA; Pub. L. No. 102-571, 106 Stat. 4491) has resulted in a substantial reduction in approval times for new molecular entities (NMEs), particularly for

Y. Richard Wang is also affiliated with the Leonard David Institute of Health Economics at the University of Pennsylvania. This study was supported in part by AstraZeneca Pharmaceuticals LP. However, the views expressed in this article are those of the authors and do not represent those of AstraZeneca Pharmaceuticals LP. The design of the analysis and interpretation of the results were performed independently by the authors. We wish to thank Peter Bogetti for help with the Spontaneous Reporting System/Adverse Event Reporting System data extraction and technical support; Adrian Gottschalk for sharing the Food and Drug Administration's new molecular entity approval data; Ernie Berndt, the editors, and an anonymous reviewer for helpful comments on an earlier draft; and Lisa Croll for administrative support.

[Journal of Law and Economics, vol. 51 (May 2008)]

(C) 2008 by The University of Chicago. All rights reserved. 0022-2186/2008/5102-0014\$10.00 
novel drugs receiving the Food and Drug Administration's (FDA's) priority rating (Berndt et al. 2005). However, the withdrawal of Vioxx and some other highprofile drug products in recent years has focused attention on whether faster reviews have adverse consequences.

Previous work offers mixed evidence on the question of whether faster reviews have negatively affected drug safety. In a series of studies, Olson (2002, 2004, 2008) found that the reductions in FDA review times were associated with increases in serious adverse drug reactions (ADRs), including those resulting in death or hospitalization. ${ }^{1}$ She also found that novel drugs receiving priority review by the FDA posed a greater risk of serious ADRs, compared with standard drugs in the same time period. In contrast, Berndt et al. (2005) found that the proportion of NME withdrawals remained unchanged in the periods before and after the implementation of PDUFA in 1992. Similar findings on new-drug withdrawals were reported by the FDA's Center for Drug Evaluation and Research (Food and Drug Administration 2005) and the Tufts Center for the Study of Drug Development (2005).

From a conceptual standpoint, PDUFA's impacts on drug safety are subject to countervailing effects. On the one hand, the FDA's staff and resources increased with the introduction of user fees under PDUFA. On the other hand, the increased speed of FDA approval under PDUFA (Berndt et al. 2005) may have introduced unknown safety risks. Furthermore, the United States is less likely to benefit from postmarketing experiences of other countries now that there has been a reversal of the drug lag phenomenon that characterized the pre-PDUFA period (Grabowski and Wang 2006).

In this article, we examine the role of faster reviews and several other explanatory variables influencing drug safety. Our analysis utilizes a negative-binomial regression model for the new drugs introduced over the period 1992-2002. The dependent variable is the number of serious adverse events reported after a product's introduction, including those resulting in hospitalization and death. Explanatory variables include FDA review times, drug novelty, global launch lags (that is, the number of months a drug is available prior to its introduction in the United States), and whether a black-box warning is required at the time of the approval. Other explanatory variables include drug utilization, the nature of the patient population, therapeutic class, mode of delivery, and time on the market.

This article is organized as follows: The next section discusses the evolution of the FDA's approach to drug safety and the implementation of PDUFA. Sections 3 and 4 describe our principal hypotheses and the data and methods. Section 5 presents the results from the negative-binomial regression model. The final section discusses our findings and their policy implications.

${ }^{1}$ Rudholm (2004) found a similar negative relationship between approval times and the number of adverse events for a small sample of new-drug introductions in Sweden between 1972 and 1996. 


\section{Historical Development of Food and Drug Administration Regulation}

\subsection{The Food and Drug Administration Regulatory Process and Issues}

The submission of statistical evidence on safety and efficacy obtained from randomized controlled clinical trials has long been the cornerstone of the drug regulatory process. ${ }^{2}$ Clinical testing normally occurs over three distinct phases, each of which offers different information about efficacy and safety. The final clinical development phase (phase 3) involves large-scale human trials, and these represent the pivotal studies of the drug's efficacy. Phase 3 studies are designed with sufficient statistical power (that is, with large enough groups of test and control subjects) to validate the preliminary evidence of efficacy developed in phase 2 .

Because several thousand individuals are commonly enrolled in phase 3 trials, testing is also useful in detecting infrequent adverse reactions. However, at this level of exposure, clinical trials normally would not detect events that occur in as few as one per 1,000 patients. Furthermore, significant interactions with other drugs and medicines could go unobserved in clinical trials, since they are done under highly controlled conditions with selective patient populations. The regulatory process relies on postmarketing surveillance to detect rare or complex interactions that are undetectable in phase 3 trials.

All drugs involve a benefit-to-risk evaluation, and regulators must make explicit or implicit trade-offs on this score in deciding when enough evidence is available to approve a drug. Historically, U.S. drug regulation has been characterized by risk-averse behavior and very long development and regulatory review times. Several researchers have argued that the FDA's risk aversion stems from the disproportionate costs regulators bear from making a type I error (that is, approving a drug that later needs to be recalled on safety grounds) compared with those involved with making a type II error (that is, not approving or unnecessarily delaying access to a drug whose benefits exceed the risks for patients). ${ }^{3}$ The latter error is less transparent, and it does not have the same damaging effects on a regulator's professional reputation.

There is a lengthy literature on how the FDA regulation of pharmaceuticals affects research and development times and costs (for a recent review, see Danzon and Keuffel 2007). A historical manifestation of the FDA's risk-averse behavior was a significant lag in the introduction of NMEs in the United States relative to the time of their introduction in other advanced economies (Kaitin and Brown 1995). DiMasi (1995) found that, by the late 1980s, the typical new drug took

\footnotetext{
${ }^{2}$ The new-drug development paradigm has been discussed in detail elsewhere (Food and Drug Administration 1999; DiMasi, Hansen, and Grabowski 2003).

${ }^{3}$ Earlier analyses of FDA regulation were focused in particular on the impact of the 1962 amendments on drug innovation to the Food, Drug, and Cosmetic Act. See, for example, Peltzman (1973), Wardell and Lasagna (1975), and Grabowski, Vernon, and Thomas (1978).
} 
more than 12 years from initial synthesis to FDA approval and that the FDA review process by itself took 2.6 years.

During the 1980s, pressures for changes in the FDA's behavior began to build as AIDS activists and other groups asked for faster access to new medicines. After a number of well-publicized events involving activists, the agency, and Congress, the FDA implemented some important initiatives targeted to lifethreatening diseases. ${ }^{4}$ First, AIDS drugs were put on a super-fast-track process for new-drug application (NDA) review (so-called AA status). Second, the FDA established in 1988 the possibility of an expedited development process for medications to treat life-threatening and seriously debilitating diseases that had no satisfactory therapeutic alternatives (the Subpart E program). ${ }^{5}$ These initiatives were followed in 1992 by accelerated approval, another regulatory program of broader scope to speed the development and approval of new agents for serious or life-threatening diseases with unmet treatment needs. In particular, the accelerated approval program allowed firms to gain approval on the basis of a surrogate end point that is reasonably likely to predict the ultimate clinical benefit (for example, time to progression of a tumor instead of long-term patient survival). The FDA could require drugs approved under this program to undergo additional postapproval clinical trials or could restrict distribution and usage to certain physician specialty groups. ${ }^{6}$

The expedited development and accelerated approval programs represented a significant departure in agency behavior. In particular, the FDA allowed speedier access and increased risk for agents to treat life-threatening and seriously debilitating illnesses on an explicit medical risk-benefit analysis. At the same time, these programs were targeted to a relatively small number of disease categoriesnotably, AIDS and certain types of cancer.

\subsection{The Prescription Drug User Fee Act}

In 1992 Congress passed PDUFA with the objective of changing the incentives for speedier review across the full spectrum of NDAs. In addition, PDUFA introduced a new mechanism, user fees, to increase the resources to undertake that task. In particular, PDUFA required drug sponsors to pay a one-time fee for an NDA or a biological licensing application (BLA) with clinical data $(\$ 1,178$ million in fees were paid in fiscal year 2008). The contributed fees were dedicated to new-drug reviews rather than other FDA tasks (until the most recent renewal of the act in 2007).

In exchange for these user fees, the FDA agreed to review and act on NDAs

\footnotetext{
${ }^{4}$ See, for example, the discussion of various initiatives targeted to AIDS and other life-threatening disease categories in Shulman and Brown (1995).

${ }^{5}$ This program allowed approval to be granted at the earliest phase of development at which safety and efficacy can be reasonably inferred. This could be done, in some cases, on the basis of a single phase 2 trial involving hundreds rather than thousands of patients.

${ }^{6}$ In 1997 the expedited development and accelerated approval programs were consolidated into the FDA's Fast Track program (Tufts Center for the Study of Drug Development 2001).
} 
and BLAs within a specified time period. In particular, NDAs and BLAs are assigned a standard or priority status at the time of submission, depending in part on the FDA's assessment of the novelty of the agent and its potential therapeutic benefits. Under PDUFA, the FDA is expected to deliver a complete review of 90 percent of priority applications within 6 months. Correspondingly, the FDA is expected to review 90 percent of standard applications within 12 months (changed to 10 months under PDUFA III). In particular, on or before the action date mandated by PDUFA, the FDA is expected to issue one of three outcome letters-approved, approvable, or not approvable. After receipt of an approved outcome letter, the company has the right to market the drug. In the case of an approvable outcome letter, the NDA or BLA can be approved if certain deficiencies and issues are appropriately addressed. Finally, a not-approvable outcome letter indicates that the company has not satisfied the FDA's standards for safety and efficacy with the evidence submitted in its NDA or BLA.

Several researchers have found that passage of PDUFA has resulted in significantly shorter FDA review times. In the most comprehensive study to date, Berndt et al. (2005) found that the regulations of PDUFA significantly accelerated a downward trend in review times that was already occurring before the act's passage. In particular, they found that review times declined from 24.2 months in 1992 to 14.2 months in 2002. Using a statistical regression model, they estimated that approximately two-thirds of this decline could be attributed to PDUFA.

The original PDUFA legislation covered a 5-year period. The law was renewed in 1997, 2002, and 2007. The size of the fees has grown significantly since 1993 and, in fact, have more than doubled in the past 5 years. The latest renewal of the act in September 2007 occurred after considerable debate about whether the user-fee structure should be maintained. This renewal included some important modifications on the allocation of user fees that are discussed in Section 6. ${ }^{7}$

\section{The Food and Drug Administration Review Process, Drug Safety Analysis, and Hypotheses}

From a conceptual standpoint, the system of user fees and performance targets under PDUFA could operate to increase or decrease a drug's risk. On the one hand, critics have argued that PDUFA has caused FDA reviewers to undertake more risks in order to meet their performance targets. On the other hand, PDUFA also produced a large infusion of new resources to evaluate a drug's benefits and risks in a timely manner.

The potential impact on drug risks from these countervailing forces is illustrated in Figure 1. In particular, one can model the potential incentive effects of PDUFA on the FDA's willingness to undertake more risks as a leftward

\footnotetext{
${ }^{7}$ See Food and Drug Administration Amendments Act, H.R. 3580, 110th Cong., 1st Sess. (2007) (http://www.fda.gov/oc/initiatives/HR3580.pdf).
} 


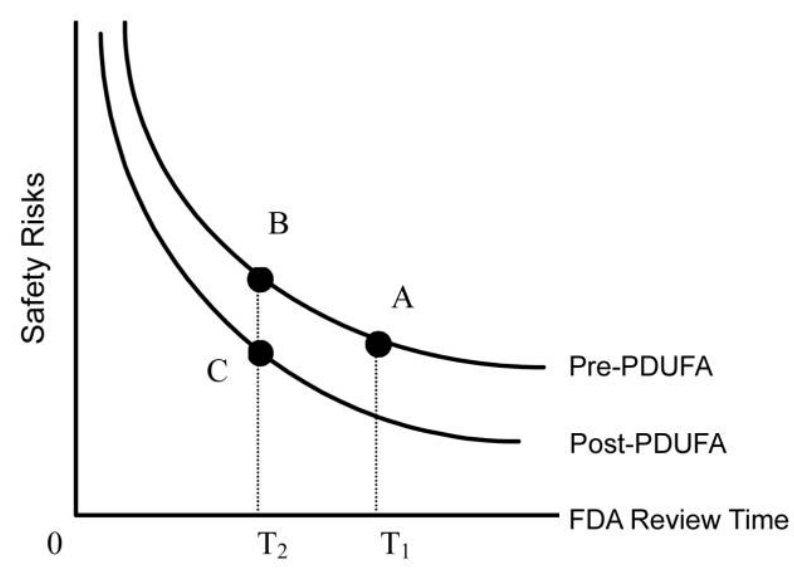

Figure 1. Trade-offs between drug safety risks and review times

movement along a negatively sloped risk-review time trade-off curve (A to B, moving from time 1 to time 2 in Figure 1). At the same time, PDUFA also effected a downward shift in the curve as a result of the increased resources from user fees (B to $\mathrm{C}$ in Figure 1). Determining the effects of shorter review times from PDUFA on drug safety is, therefore, an empirical issue.

A number of other regulatory-related factors besides review times also affect drug safety. Food and Drug Administration approval decisions are based primarily on data from controlled clinical trials undertaken by the applicant under FDA guidelines. The size and duration of these trials, therefore, can have an important effect on the FDA's ability to discern risks before approval. The average number of clinical trial patients for approved NMEs has grown significantly since the early 1990s. This increases causes the risk-return curve in Figure 1 to shift downward over time. ${ }^{8}$

The primary objective of this study is to understand how speedier reviews under PDUFA have affected drug safety, taking account of other potential confounding factors. The remainder of Section 3 is focused on regulatory-related variables, while other control variables are discussed in Section 4.

\subsection{Introductions Abroad prior to Those in the United States}

While clinical trial sizes have been increasing, the identification of rare but serious side effects (that is, risks involving one or fewer incidents in 1,000 patients) generally requires information based on postmarketing experience. Prior to the mid-1990s, most new drugs were introduced into Europe and other

${ }^{8}$ See in particular the analysis of trends in this regard in DiMasi, Hansen, and Grabowski (2003, p. 177, n. 41). In particular, the mean number of subjects for new-drug applications in 1998-2001 exceeded 5,000 compared to just over 3,000 at the start of the 1990s. 
countries before entering the United States. This advance introduction provided spillover knowledge inputs to U.S. regulators. This process has changed in recent years, owing to both shorter regulatory reviews in the United States and the movement to harmonize regulatory procedures between the United States and the European Union.

The fact that many commercially important new drugs are now introduced first in the United States, or simultaneously in other countries, results in a shift upward of the risk-review time curve shown in Figure 1. In assessing the level of risks associated with review times pre- and post-PDUFA, it is therefore important to consider whether a drug has been marketed outside the United States, and for what length of time, prior to U.S. approval. This is one of the control variables included in our empirical analysis.

\subsection{Drug Novelty}

Novel drugs are also expected to incur above-average risks. As was discussed, the Subpart E and accelerated approval programs that began in the 1990s target drug treatments for serious and/or life-threatening diseases with large unmet medical burdens. These programs, by design, allow for expedited development schedules and approval based on surrogate end points. The FDA, in effect, has made a policy decision to accept greater risks to speed the availability of new drugs with large potential benefits in areas of unmet medical need. Consequently, it is reasonable to expect higher postlaunch risks for drugs that meet the criteria for these programs.

Priority drugs are another class of specially designated drugs by the FDA. As we discussed, when a firm submits an NDA (or BLA) to the FDA for review, the drug is classified as priority or standard. Priority drugs go to the head of the queue in terms of FDA resources and have the shortest review time targets6 months under PDUFA. According to the Center for Drug Evaluation and Research, a priority designation is given to products that have the likelihood of providing a significant therapeutic advance, compared with marketed products, because of increased effectiveness, safety, or patient tolerability and compliance. ${ }^{9}$ Since many priority drugs rely on a novel mechanism of action, they are also subject to greater uncertainty about safety risks. Consequently, it is reasonable to expect higher risk levels for priority drugs.

In addition to first-order effects, there may also be interactive effects between PDUFA and the novel drug classes. In particular, given the pressures associated with PDUFA for faster access to novel drugs, these agents may be subject to greater relative risks after its passage than are standard drugs. This hypothesis is also considered in our empirical analysis.

${ }^{9}$ The FDA guidelines for a priority review are set forth in U.S. Food and Drug Administration, Center for Drug Evaluation and Research, Manual of Policies and Procedures, chap. 6020.3, pp. 1-2 (http://www.fda.gov/Cder/mapp.htm). 


\subsection{New Biological Entities}

Biological drugs are another class of novel agents that have grown in importance since the early 1990s. Most biologicals were reviewed by the Center for Biological Evaluation and Research (CBER) during the $1992-2002$ period. ${ }^{10}$ In contrast to the criteria for new chemical entities, new biological entities receive priority review by the CBER only when they offer the potential of a significant therapeutic advance for life-threatening diseases or conditions. ${ }^{11}$ In a recent article, we found that new biological entities are focused particularly on treatment for cancer and other life-threatening diseases (Grabowski and Wang 2006). We also found that new biologicals have accounted for a disproportionate share of first-in-class drugs since 1993. Given these characteristics, we expect biologicals also to be subject to above-average risks. ${ }^{12}$

\subsection{Black-Box Warnings and Anticipated versus Unanticipated Risks}

An important distinction in FDA decision making is between known (or anticipated) risks and unknown (or unanticipated) risks. Through a product's labeling at the time of approval, the FDA can signal a known risk of a serious nature to physicians and patients. In particular, when the FDA decides to approve a new drug for which the expected therapeutic benefits outweigh a known safety risk of a serious nature, it can require a black-box warning on the initial label. An example of such a case is the drug Herceptin. This drug was approved by the FDA in 1998 after a review time of slightly less than 5 months. Herceptin constitutes an important advance in the treatment of breast cancer patients that express excessive HER-2 protein. However, treatment with Herceptin also significantly increases the risk of congestive heart failure (see Herbst et al. 2006). ${ }^{13}$ Consequently, the drug was given both a priority review and a black-box warning at the time of its initial approval.

It is reasonable to postulate that drugs that receive a black-box warning at initial approval will be subject to above-average risks after launch. A situation,

\footnotetext{
${ }^{10}$ The main exceptions were some of the initial biological products that were approved for marketing by the Center for Drug Evaluation and Research, such as recombinant insulin and human growth hormone. Recently, the center has assumed increased responsibilities for reviewing new biological entities but will maintain the same criteria for approval on these entities that were employed by the Center for Biological Evaluation and Research (CBER).

${ }^{11}$ U.S. Food and Drug Administration, Center for Drug Evaluation and Research, Manual of Policies and Procedures, chap. 6020.3, p. 2 (http://www.fda.gov/Cder/mapp.htm).

${ }^{12}$ Some classes of biological drugs have higher risks than others do-for example, compare monoclonal antibodies with recombinant products. This difference is reflected in the black-box warning variable discussed below. In particular, five of the nine monoclonal antibody products (56 percent) carry a black-box warning at approval. This compares to five of the 24 recombinant products ( 21 percent).

${ }^{13}$ For breast cancer patients with excessive amounts of HER-2 protein (25-30 percent of patients), Herceptin cuts the risk of recurrence by half but also increases the risk of congestive heart failure (the incidence of severe congestive heart failure or death from heart problems after 3 years in the first two trials was 2.9-4.1 percent for women taking Herceptin versus .0-.8 percent for the control group).
} 
however, in which this might not be the case involves black-box warnings targeted to particular subpopulations for whom the drug is contraindicated (for example, pregnant women). For example, the class of angiotensin-converting enzyme inhibitors/angiotensin receptor blockers are widely utilized drug treatments for hypertension. The complete class carries a black-box warning for pregnant women in their second and third trimesters on these drugs' increased risk of causing birth defects. If this type of black-box warning is effective in causing pregnant women to substitute other antihypertensive treatment regimes, the warning would not necessarily be associated with above-average risks after this type of drug's launch.

\subsection{Summary of Major Hypotheses}

In our empirical work, we investigate what variables have affected the incidence of serious ADRs associated with new drugs introduced between 1992 and 2002. With respect to the regulatory-related variables, we focus on the following hypotheses:

1. Novel drugs, that is, those subject to accelerated approval or priority review, are expected to be riskier to patients.

2. Biological drugs are expected to be riskier on the basis of both novelty and an emphasis on treating conditions with high disease burdens and unmet medical needs.

3. Drugs subject to initial black-box warnings generally involve cases of high benefits and risks. Therefore, they are expected to have more adverse events after approval, unless the black-box warning is directed to a particular subpopulation or demographic group for which the drug is contraindicated.

4. Knowledge spillovers associated with foreign marketing experiences are expected to reduce safety risks in cases where U.S. launch lags exist, especially when data become available from a large population abroad over an extended period prior to U.S. approval. In other words, longer U.S. lags should result in fewer adverse events.

5. After controlling for these and other determinant factors, shorter FDA review times under PDUFA may or may not be associated with greater safety risks, given the countervailing effects of PDUFA presented above. In addition, potential interactive effects between PDUFA and riskiness of the various categories of novel drugs are considered in the analysis that follows.

\section{Methodology and Data}

\subsection{Model Specification}

The negative-binomial model is typically employed when the dependent variable involves counts that are overdispersed and do not fit the Poisson distribution (Cameron and Trivedi 2005). Given that this is the case here, we employ the 
negative-binomial model to model the occurrence of ADRs for NMEs introduced between 1992 and 2002..$^{14}$

The basic specification for the negative-binomial regression model in the current analysis is

$$
E\left(\mathrm{ADR}_{i t}\right)=\exp \left(\alpha R_{i t}+\beta N_{i t}+\gamma P_{i t}+\varepsilon_{i t}\right)
$$

where $E\left(\mathrm{ADR}_{i t}\right)$ is the expected value of the counts of ADRs, $R_{i t}$ are the regulatory-related variables, $N_{i t}$ are the NME characteristic variables, and $P_{i t}$ are the patient-specific variables. Separate models are estimated for serious ADRs, hospitalization ADRs, and death ADRs. The data sources and the definitions of all the explanatory variables are discussed in the next two sections.

\subsection{Adverse Drug Reactions}

The dependent variable in equation (1) is the count of ADRs in the year of launch and the first 2 years after launch. We identified the annual number of adverse events for 1992-2002 NMEs using the Spontaneous Reporting System (SRS) and Adverse Event Reporting System (AERS) databases maintained by the FDA (Rodriguez, Staffa, and Graham 2001; Wysowski and Swartz 2005). ${ }^{15}$ The ADRs listed in these combined databases are voluntary, spontaneous reports filed primarily by health care professionals such as clinicians, physicians, and pharmacists. We focused in particular on serious ADRs, defined as those resulting in hospitalization, death, or disability or determined to be life threatening or require intervention to prevent permanent impairment or damage.

A strength of these databases is their broad coverage of all patient populations and all therapeutic uses, including approved indications and possible off-label use. They are particularly useful for detecting rare ADRs that are not captured in clinical trials and for detecting drug-drug interactions. The ADRs reported in a drug's initial years on the market are the main basis for revisions in a drug's label, including new warnings and contraindications. In extreme cases these ADR reports can also result in a product's withdrawal.

Some of the recognized limitations of the ADR databases are their underreporting of ADRs, the uncertainty about causation, and the fact that there is no risk adjustment for utilization and other factors. We structured our analysis in various ways to deal with these different concerns. First, with respect to the underreporting issue, we focused on an NME's annual number of serious ADRs within the first 3 years of marketing. The reporting of adverse events is highest

\footnotetext{
${ }^{14}$ The negative-binomial model has been utilized in the analysis of adverse drug events by Olson (2002, 2004, 2008) and Rudholm (2004).

${ }^{15}$ The Spontaneous Reporting System (SRS) database, which was started in 1969, was later replaced by the Adverse Event Reporting System (AERS) database in November 1997. Our SRS/AERS data were provided by Lincoln Technologies and ended in the third quarter of 2004. In both the SRS and AERS databases, adverse drug reactions (ADRs) could be reported under either the brand or the generic name. Therefore, using only the brand name to count ADRs potentially underestimates the number of adverse events. Lincoln Technologies standardized the generic name for each reported drug in its data releases, thereby making it easy to count all ADRs associated with a particular drug.
} 
in the first 2 years after approval (Rodriguez, Staffa, and Graham 2001; Lasser et al. 2002). ${ }^{16}$ With respect to the causation issue, we considered only ADRs for drugs suspected to be the cause. ${ }^{17}$

We address concerns about the role of drug exposure and other factors affecting ADR reports by employing a multivariate regression framework described in equation (1). In particular, the set of determinant variables include a drug's exposure in terms of physical unit sales, whether the drug is a biological or chemical entity, its product formulation (oral, injectable, or other), its therapeutic class, its patient user characteristics, and so forth. These determinant variables are discussed in detail in the sections that follow.

\subsection{The New Molecular Entity Sample}

There were 328 new biological and new chemical entities approved between 1992 and 2002. This information is taken from the FDA database of NME approvals. Our analysis specifically excludes NMEs classified as vaccines, diagnostic agents, radiopharmaceuticals, over-the-counter products, combination products, or any new formulations of existing products over the 1992-2002 period. In addition, 35 NMEs were excluded on the grounds of missing information in the SRS/AERS databases or in one of the other databases used to construct the explanatory variables in the analysis. ${ }^{18}$ Our final sample consisted of 293 unique NMEs and 823 NME-year observations.

We focused on NMEs approved between 1992 and 2002 on the basis of the time-series information available in the different databases. We excluded NMEs approved prior to 1992 because our sales volume data (used to adjust for patient exposure) started in 1993. Since adverse-event reporting is highest in the first 2 years after approval, it was not feasible to include NMEs approved before 1992 . Correspondingly, our last year with complete data on adverse events was 2003, so we excluded all NMEs approved after 2002.

Our sample includes the withdrawn NMEs that were approved between 1992

\footnotetext{
${ }^{16}$ We could not use smaller time units such as a quarter because the event-month variable is missing for a significant portion of the ADRs. We excluded foreign and study ADRs, as the former were for non-U.S. patients and the latter were for ADRs that occurred in postmarketing clinical studies (and therefore were not related to product consumption or commercial sales in the United States).

${ }^{17}$ However, in contrast to Olson (2008), for an ADR possibly associated with more than one drug, we assigned an equal $1 / N$ share of the ADR to each suspected drug. Note that when the AERS replaced the SRS in October 1997, the AERS added the distinction of a primary suspected drug when there were multiple suspected drugs involved in a particular ADR. Prior to that time, all suspected drugs were given equal treatment in the listings in the SRS database. To be consistent over time, we divided all suspected drugs by $1 / N$, whether they are classified as primary or secondary suspected drugs after October 1997. By contrast, Olson's approach is subject to multiple drug counts for all her observations under the SRS system.

${ }^{18}$ Of the 35 NMEs excluded because of missing data, there are six products without a unique generic name in the SRS/AERS databases, 12 products that are missing, 11 products without sales volume information, four products belonging to two therapeutic classes without patient demographic information, one product withdrawn in 1992, and one product without a priority or standard review designation.
} 
and 2002. The lone exception is Omniflox, which was approved and withdrawn in 1992 (and therefore was excluded because of missing sales data and an incomplete ADR profile). Furthermore, 54 of the 293 NMEs in our sample were approved with an initial label requiring a black-box warning.

Following the FDA, we defined PDUFA NMEs as those NDAs or BLAs submitted on or after October 1, 1992. With this definition, 18 percent of our NMEyear observations are pre-PDUFA, and the remaining 82 percent are PDUFA NME-year observations.

\subsection{Explanatory Variables}

The key explanatory variables of interest relate to the bundle of regulatory variables. Information on each drug's speed of review (the time between NDA or BLA filing and approval) was obtained from the FDA. ${ }^{19}$ We also obtained data on which NMEs received the FDA's priority or standard ratings. We obtained the list of accelerated approvals from the FDA's Web site and defined accelerated approvals as NDAs approved under Subpart H (21 C.F.R. 314.510) and BLAs approved under Subpart E (21 C.F.R. 601.42). Our study sample included 27 accelerated approvals. ${ }^{20}$

Data on whether an NME's label required a black-box warning at the time of approval were obtained from the FDA's Web site and annual editions of the Physician's Desk Reference. ${ }^{21}$ Furthermore, the warning labels were analyzed as to whether they involved restrictions on a specific subpopulation of patients. In particular, 11 of the 54 NMEs with black-box warnings at the time of approval were contraindicated for pregnant women owing to the risk of birth defects but carried no black-box warnings for the general population. These NMEs were treated as a separate category from the other NMEs with black-box warnings. The more general category of black-box warnings consisted largely of NMEs for life-threatening diseases and conditions, especially cancer $(n=15)$ and AIDS $(n=11)$. The 11 drugs contraindicated for pregnancy included nine antihypertensive agents, one oncology drug, and one drug for rheumatoid arthritis.

We used the MIDAS database provided by IMS Health ${ }^{22}$ to obtain information on a drug's first worldwide launch date, formulation (oral, injectable, or other), therapeutic class, and sales volume. The therapeutic class was defined as the

\footnotetext{
${ }^{19}$ For the approval times, see U.S. Food and Drug Administration, Center for Drug Evaluation and Research, CDER Drug and Biologic Approval Reports (http://www.fda.gov/cder/rdmt/).

${ }^{20}$ The expedited development program (Subpart E for NMEs) was rarely used after the implementation of PDUFA, and, to the best of our knowledge, the full list of expedited development approvals has not been made available by the FDA. Initially, however, it was not uncommon for NMEs to be jointly covered under both the accelerated approval and expedited development programs (Shulman and Brown 1995). After 1997, both programs were incorporated into the Fast Track program.

${ }^{21}$ Two other sources were utilized as a check on our data for black-box warning. Joyce Generali has compiled a comprehensive list of drugs with black-box warnings (FormWeb, Black Box Warnings [http://www.formularyproduction.com/blackbox]). Also, Lasser et al. (2002) examined the timing and addition of black-box warnings for all new chemical entities approved between 1975 and 1999.

${ }^{22}$ For information on the MIDAS database, see http://www.imshealth.com.
} 
four-level Anatomical Therapeutic Classification (ATC) code, developed and maintained by the European Pharmaceutical Marketing Research Association. ${ }^{23}$ This information from MIDAS was used to construct a U.S. launch lag variable (that is, the elapsed time from an NME's first launch worldwide).

We also used data from MIDAS to estimate the annual U.S. sales volume for the combined retail and hospital channels for each NME. The MIDAS database reports quarterly sales volumes in standard units (SU). This is defined by IMS Health, for example, as one tablet, one capsule, 5 milliliters of oral liquid, one ampoule, and so forth. ${ }^{24}$ We constructed separate sales measures for oral, injectable, and other forms, given that the units of measurement are different for these forms. As injectable products are mainly consumed in the hospital setting, it is important that we include the hospital channel to accurately measure patient exposure to these products. ${ }^{25}$

Finally, we used the 2003 National Disease Therapeutic Index (NDTI) database, also provided by IMS Health ${ }^{26}$ to estimate the age and sex distribution of patients treated by an NME. The NDTI is a nationally representative survey of ambulatory medical care visits in the United States. Using 2003 NDTI data, we estimated for a certain therapeutic class the percentages of patients treated who were under 20 years of age, who were at or above 65 years of age, and who were female. These therapeutic-class-level variables were used as proxies to reflect the demographic characteristics of patients treated by an NME.

\section{Results}

\subsection{Summary Statistics}

As we mentioned before, our final sample consisted of 293 NMEs including 12 withdrawn products that were approved between 1992 and 2002. Figure 2 presents the distribution of these NMEs by year of approval and also by withdrawal status, with the most approvals in $1996(n=47)$ and the most withdrawals in $1997(n=5)$. Figure 3 shows that these NMEs belong to 14 therapeutic areas (the first level of ATC classification). ${ }^{27}$ The greatest numbers of

${ }^{23}$ For information on the Anatomical Therapeutic Classification code, see European Pharmacuetical Market Research Association, Anatomical Classification (http://www.ephmra.org/main.asp?page $=465$ ).

${ }^{24}$ Our approach differs from that of Olson (2008), who used the number of retail prescriptions based on the Medical Expenditure Panel Surveys (MEPS) beginning in the year 1996.

${ }^{25}$ We have access to another IMS Health database, NPA Plus, for the year 2003 (http://www imshealth.com). The NPA Plus database reports the number of prescriptions dispensed through retail pharmacies in a given year. For the 275 NMEs that we could match with NPA Plus data in 2003, the correlation ratio between our defined sales volume in standard units (SUs) and NPA Plus-reported number of retail prescriptions is .77 $(p<.0001)$.

${ }^{26}$ For information on the NDTI database, see http://www.imshealth.com.

${ }^{27}$ The following abbreviations were used in Figure 3: $\mathrm{A}=$ alimentary tract and metabolism, $\mathrm{B}=$ blood and blood-forming organs, $\mathrm{C}=$ cardiovascular system, $\mathrm{D}=$ dermatologicals, $\mathrm{G}=$ genitalurinary system and sex hormones, $\mathrm{H}=$ hormone therapy, $\mathrm{J}=$ systemic anti-infectives, $\mathrm{L}=$ oncology, $\mathrm{M}=$ musculoskeletal system, $\mathrm{N}=$ central nervous system, $\mathrm{P}=$ parasitology, $\mathrm{R}=$ respiratory system, $\mathrm{S}=$ sensory system, and $\mathrm{V}=$ various others. 


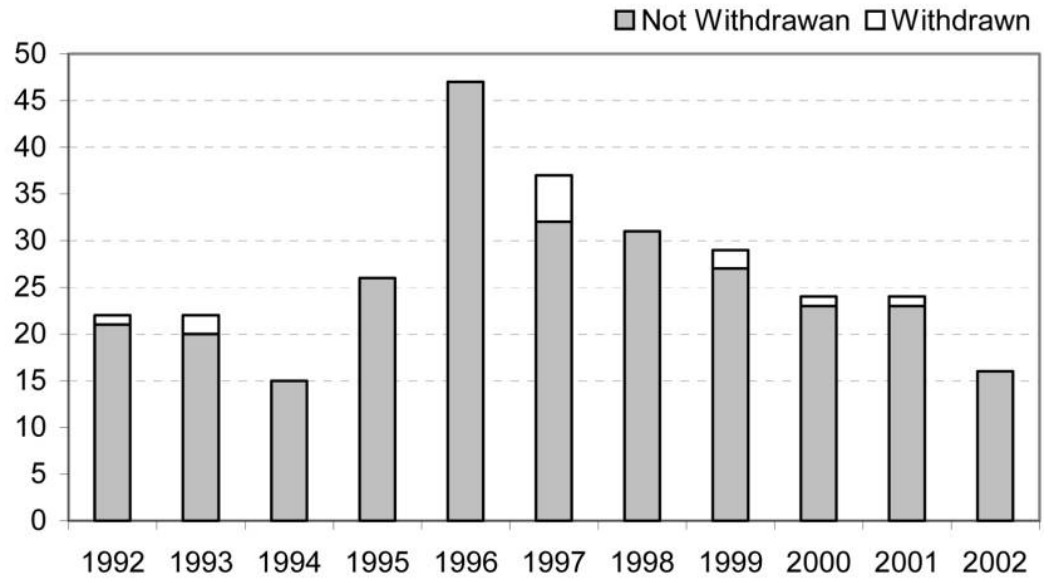

Figure 2. Distribution of sample new molecular entities by year of Food and Drug Administration approval.

products come from the central nervous system $(n=49)$, oncology $(n=45)$, and systemic anti-infective $(n=41)$ categories. Of the 293 NMEs, 31 were biological products and 44 were orphan drugs.

Variable definitions and characteristics of the $823 \mathrm{NME}$-year observations are presented in Table 1. Noticeably, the distributions of ADRs, FDA review time, U.S. launch lag, and sales volume are highly skewed. In the negative-binomial models, we used natural log specifications for FDA review time and U.S. launch lag. ${ }^{28}$ This log transformation was used because the distributions are highly right skewed and also because we expect their marginal effects to decrease with time. In addition, the sales volume variables for oral, injectable, and other formulations were specified as natural logs owing to the skewed distribution.

Prescription Drug User Fee Act NMEs account for 82 percent of the observations. Priority drugs, accelerated approvals, and biological products account for 42 percent, 9 percent, and 10 percent of the observations, respectively. The predominant formulation is oral, the next most common is injectable, and last is others. Note that the product age is defined as the number of full calendar years after launch, with the year of launch designated as zero. Also note that the year of launch may differ from the year of approval.

For the results shown in Table 2, we used data from the first year of positive sales to compare characteristics of standard NMEs, priority NMEs, and NMEs with accelerated approval. ${ }^{29}$ In this table (but not the others), the three categories

\footnotetext{
${ }^{28}$ We added one to all observations for the therapeutic class lag and U.S. launch lag to avoid the log-of-zero problem.

${ }^{29}$ For the 12 NMEs launched in 1992, the first available sales data were from 1993. In these cases, data for 1993 were used to calculate the values in Table 2.
} 


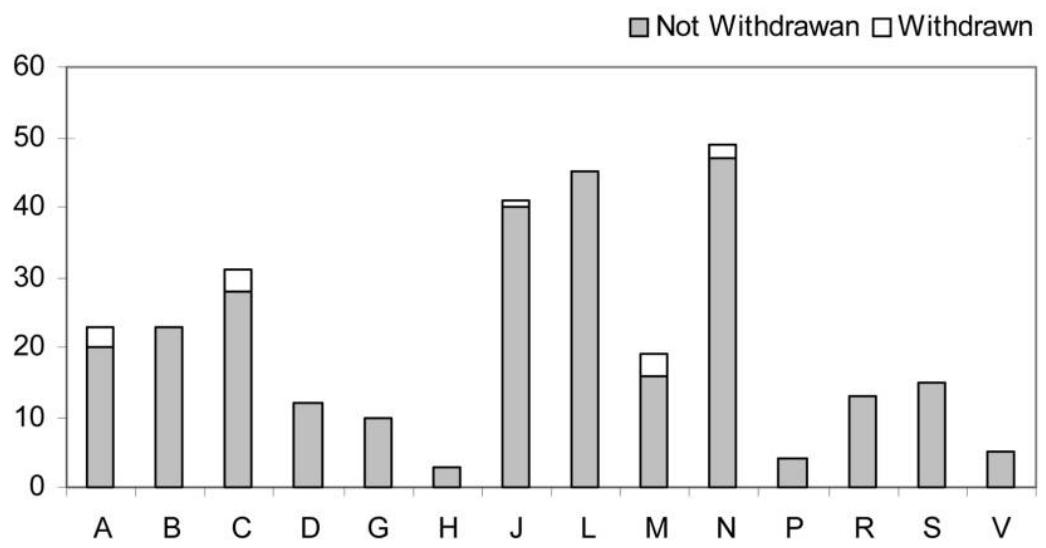

Figure 3. Distribution of sample new molecular entities by therapeutic area

are mutually exclusive, because the 27 NMEs with accelerated approval-25 priority NMEs and two standard NMEs-are not included in the other categories. ${ }^{30}$ Priority drugs have the highest sales volumes, the next highest are for standard drugs, and last are accelerated approvals. Adjusted for sales volume, accelerated approvals have the highest number of ADRs per thousands of SUs, priority drugs have the next highest, and standard drugs have the least. Table 2 also shows that the percentages of biological and orphan products increase for priority products and accelerated approvals. The mean FDA review time decreases for priority products and accelerated approvals. Because of a few outliers, the mean U.S. launch lag is the longest for priority drugs. By contrast, the median U.S. launch lag is 3 months for standard drugs and 0 months for priority drugs and accelerated approvals.

The group of drugs with black-box warning labels at the time of initial approval is disproportionately concentrated in the priority and accelerated approval categories. Only 5 percent of standard approvals receive these black-box warnings. By contrast, 24 percent of priority drugs and 44 percent of accelerated approvals receive black-box warnings. This option is therefore utilized primarily for novel drugs with large expected therapeutic benefits. The pattern of black-box warnings is very different for the special group involving contraindications for pregnant women. The vast majority of these drugs are standard drugs.

We also separated the NME data shown in Table 2 into observations for the pre-PDUFA and post-PDUFA periods. As expected, the major differences between the two samples are in the FDA review times. In particular, the mean FDA review times for standard NMEs was 43 months before passage of PDUFA

${ }^{30}$ This division into mutually exclusive categories is only for comparative purposes. When constructing the variables in the regression analyses (Tables $1,3-5$ ), the NMEs with accelerated approval are also included in their appropriate priority or standard review categories. 


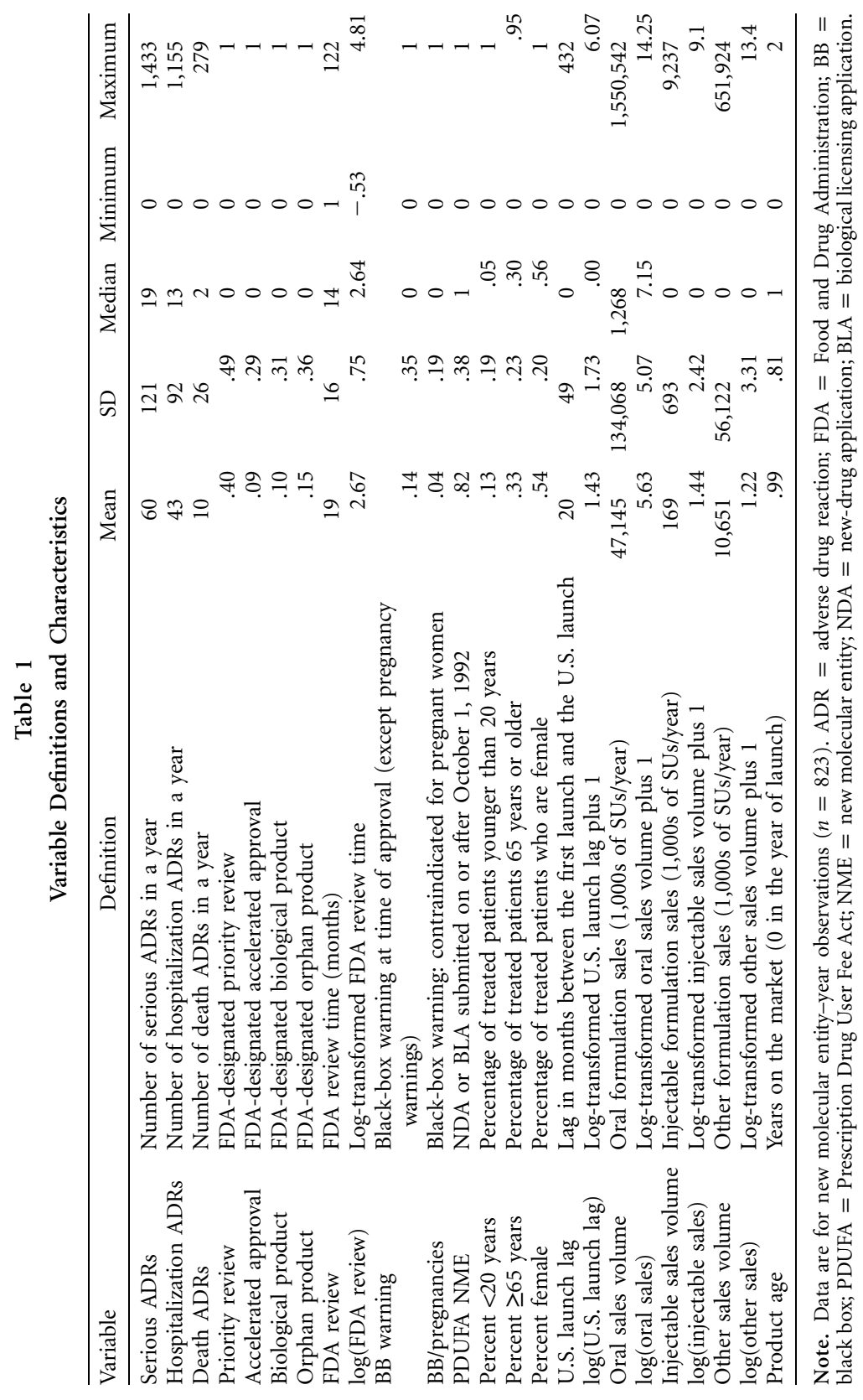




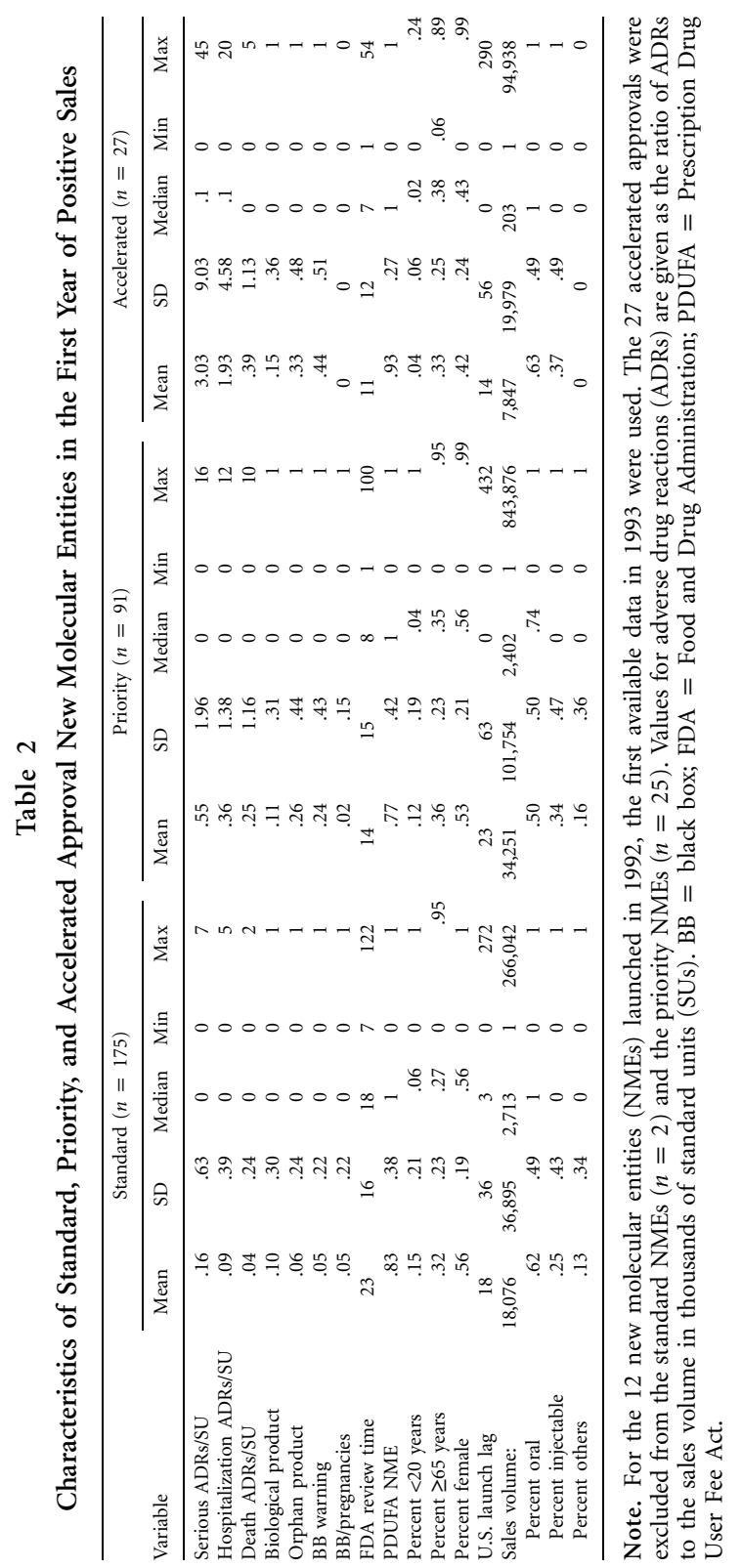


$(n=30)$ and 19 months after passage of PDUFA $(n=145)$. The mean review times for priority NMEs was 29 months before PDUFA $(n=21)$ and 10 months after PDUFA $(n=70)$. The mean values for accelerated approvals were similar to those for priority drugs -26 months before PDUFA $(n=2)$ and 10 months after PDUFA $(n=25)$. Hence, novel drugs not only experienced shorter review times compared to those for standard drugs but also had a greater percentage decline for the post-PDUFA period relative to the pre-PDUFA period.

\subsection{The Negative-Binomial Regression Model}

We present the negative-binomial model estimates for the full sample of NMEyear observations in Table 3. We utilize the maximum likelihood estimating procedure, with adjustments for multiple observations from the same NME. ${ }^{31}$ We estimate separate regression models for serious, hospitalization, and death ADRs. The results in Table 3 are generally consistent for each category of ADR, but the dispersion coefficient is substantially higher for the death ADR equations than for the serious ADR or hospitalization ADR equations. ${ }^{32}$ This finding apparently reflects the fact that death ADRs exhibit a more skewed distribution (Table 1) and also have more zero counts. ${ }^{33}$

As we hypothesized, more novel drugs, including priority products, those with accelerated approvals, and biological products, have more associated serious adverse events. For these indicator variables, the marginal effect is the coefficient times the mean of the dependent variable. For example, the mean is 60 for serious ADRs in Table 1. Therefore, ceteris paribus, a priority product leads to 57 additional serious ADRs annually, an accelerated approval product leads to 35 additional serious ADRs, and a biological product leads to 75 additional serious ADRs. In contrast, orphan products are not associated with additional serious ADRs in any of the specifications. ${ }^{34}$

We estimated both a log and a linear variant of the FDA review time variable. Given the skewed nature of the distribution of review times, a logarithmic specification was the preferred variant utilized in the basic model. The alternative model contains the linear specification on review times for comparative purposes. While these coefficients were negative, they were statistically insignificant for all

\footnotetext{
${ }^{31}$ We used the GENMOD procedure in SAS version 8.1, with the REPEATED statement to adjust for multiple observations from the same NME over time, to estimate the negative-binomial models. Because the biological products in our study sample were approved by the former CBER, we also ran separate analyses for NDAs and BLAs. The results were qualitatively similar, and therefore all NMEs were combined for the data presented in Table 3.

${ }^{32}$ In the negative-binomial regression model, the variance of the distribution is a quadratic function of the mean with the coefficient on the quadratic term being denoted as the dispersion parameter (Cameron and Trivedi 2005).

${ }^{33}$ One indication of greater skewness is the fact that the ratio of the mean to the median in Table 1 is greater for death ADRs than for serious or hospitalization ADRs. In addition, for the 823 NMEyear observations, there are 81, 105, and 206 zero observations, respectively, for serious, hospitalization, and death ADRs.

${ }^{34}$ It should be noted, however, that orphan products are disproportionately represented in the priority and accelerated approval categories, which are associated with more adverse events.
} 


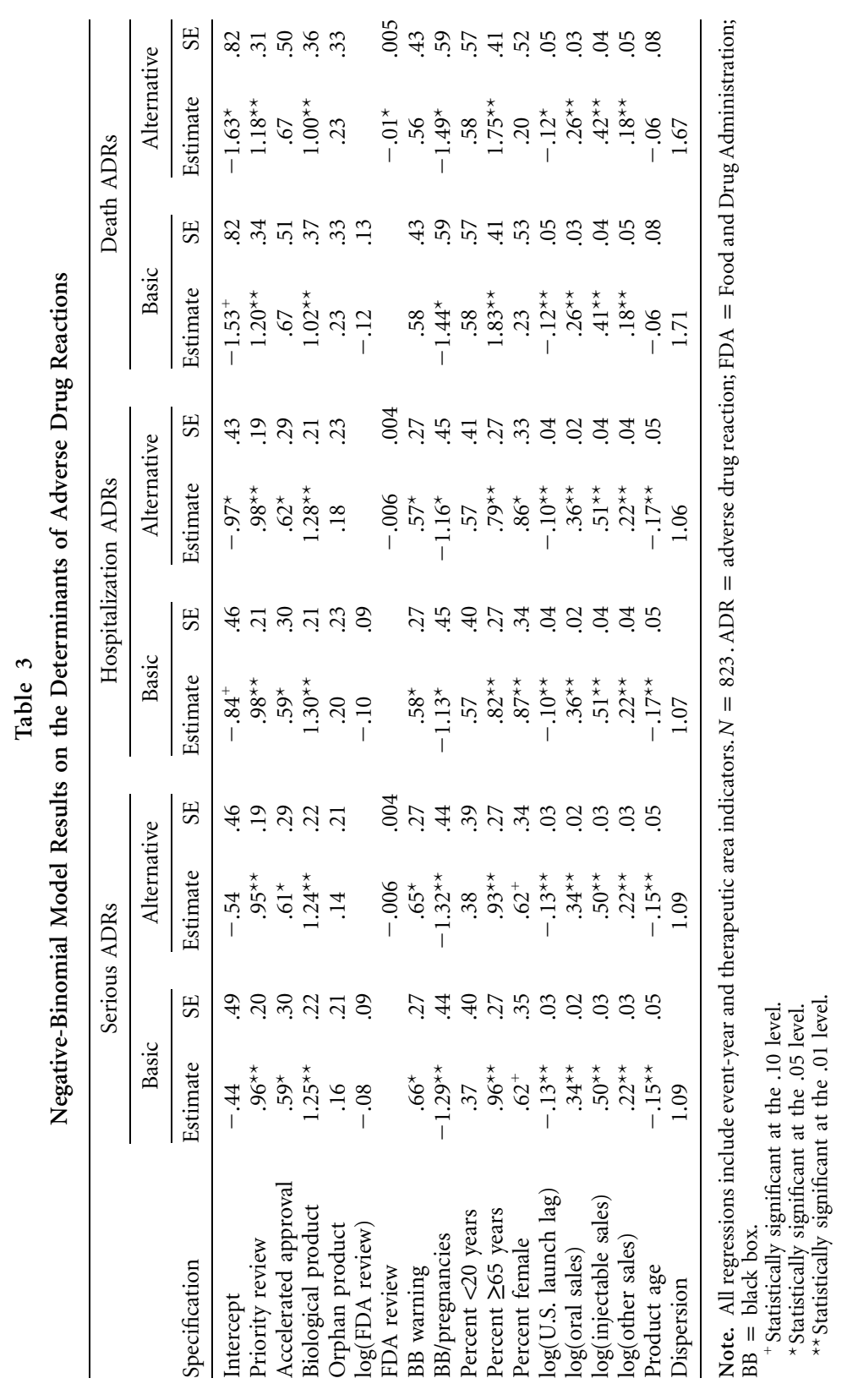


three dependent variables, and this finding indicates that shorter review times, ceteris paribus, have not led to additional serious adverse events. The coefficients for the linear formulation for review times were also statistically insignificant, except in the case of death ADRs. ${ }^{35}$

In contrast to the review time variable, the U.S. launch lag has a negative and statistically significant effect on the occurrence of serious ADRs in all specifications. In particular, ceteris paribus, if one compares the case of first launch in the United States with the situation in which the United States experiences a launch lag of 12 months, the marginal impact is 20 fewer serious adverse events annually with the lagged introduction. We also tried two variations of this variable-a population-weighted launch lag and a simple indicator variable on whether the drug was introduced abroad first. The launch lag variable and its population-weighted alternative performed comparably. The coefficients were statistically significant $(p<.05)$ in all cases. ${ }^{36}$ The simple indicator variable had a negative, but not statistically significant, coefficient, and this finding indicates that the length of the lag mattered.

The coefficients for the two black-box warning variables exhibit opposite signs. The coefficients for the general black-box warning have the expected positive sign and are statistically significant, except the one for death ADRs. By contrast, the coefficient for the black-box warning variable pertaining only to risks for pregnant women has a statistically significant negative sign. A plausible interpretation of this negative sign is that the warning appears to be successful in causing pregnant women to substitute other medications and, furthermore, that these indicated drugs have below-average relative risks when utilized by other patient populations. ${ }^{37}$

A drug's sales volume is a strong predictor of the level of its ADRs. All three product type formulations have sales coefficient estimates with $p$-values statis-

${ }^{35}$ For this latter case, the estimated coefficient on the linear review time variable implies that a 1-year reduction in review times would be associated with 1.2 more death ADRs per year. However, this observed effect may be the result of a few outlier observations, given that the coefficient for the logarithmic formulation of the review time variable is always insignificant. As we noted, death ADRs are subject to much greater dispersion than are serious or hospitalization ADRs. Furthermore, coefficients for neither the linear nor the logarithmic variants of review time in Table 3 are significant when the dependent variable is the more frequently observed serious ADR or the hospitalization ADR.

${ }^{36}$ Population weights provide only a rough measure of the number of patients exposed outside the United States, given differences across countries in utilization controls and disease burden. We used the New Product Focus database from IMS Health (http://www.imshealth.com), which tracks the launch dates of new drugs in 22 major markets worldwide since the early 1980s, to construct the population-weighted global launch lag variable for the United States (Grabowski and Wang 2006). For an NME, we first calculated the U.S. launch lag with each of the other 21 countries (the value equals zero if the U.S. launch was earlier or at the same time). We then weighted this U.S. launch lag with that country's total population in 2004, and the sum of these weighted launch lags is that NME's population-weighted global launch lag for the United States. Note that this variable is also $\log$ transformed in the regressions.

${ }^{37}$ As we discussed, nine of the 11 drugs in this group are antihypertensives for which a classwide black-box warning on pregnancy risks has been in effect for many years. Many alternative medications are also available to treat hypertension in pregnant women. 
tically significant at the 1 percent level, with the highest value observed for injectables. ${ }^{38}$ Other positive and statistically significant predictors of adverse events include drugs targeted to older patients (those 65 years or older) and to female patients. As we expected, a product's age is negatively associated with its level of reported ADRs. This finding is consistent with a downward trend in mean ADRs per unit of exposure over the first 3 years of marketing for each category of ADR. Therapeutic class and event-year indicator variables are included in all specifications but are not separately reported.

The extent to which the voluntary reporting nature of the SRS/AERS databases affects our findings is unknown. For example, physicians may be more motivated (from scientific curiosity) to report adverse events caused by novel drugs, and this situation would lead to an upward bias for novel drugs. ${ }^{39}$ Drugs dispensed by physicians in hospitals and clinics (such as injectable new biological entities) also may be subject to more ADR reporting because of the more structured institutional setting. Improved postmarketing systems for collecting ADRs, as recommended by the Institute of Medicine (2007) and other bodies, could provide insights on this issue.

\subsection{Sensitivity Analyses}

We performed a number of sensitivity analyses designed to consider interaction and other potential effects of PDUFA on drug safety. For this purpose we constructed a PDUFA indicator variable on the basis of whether the NME was submitted to the FDA after the passage of PDUFA (that is, on or after October $1,1992)$. We tried various specifications in which the PDUFA variable was interacted with the various measures of drug novelty-interactions with priority, accelerated approval, and biological drugs. None of these interaction effects were statistically significant. ${ }^{40}$ Hence, there is no evidence that PDUFA disproportionately increased the risks associated with novel drugs, despite their shorter review times.

We also tested the interactions between product age (years after FDA approval) and the three novelty variables to see whether the difference between novel products and less novel ones narrows over time. None of these interaction effects were statistically significant, and this finding indicates that novel drugs remained

\footnotetext{
${ }^{38}$ The higher coefficient observed for unit sales of injectables could reflect the higher risks for these products. Alternatively, it could be the result of differences in dosing or more adverse-event reporting for products dispensed primarily in hospitals.

${ }^{39}$ Similar to Olson $(2002,2004)$, we find that female patients are more likely to experience adverse events. While this increase could be caused by less clinical trial testing on female patients prior to a drug obtaining marketing approval, another possible explanation is that female patients are more likely to seek medical care and therefore report the adverse events.

${ }^{40}$ In addition, when the PDUFA variable was considered as an additional independent variable, with or without the review time variables present, its coefficient was always statistically insignificant. Hence there is no evidence that the implementation of PDUFA caused a regime shift toward higher risks across the spectrum of review times (that is, an upward shift of the risk-review time curve in Figure 1). It should also be noted that the coefficient estimates on the other variables remain essentially unchanged when the review time (log-linear or linear) variable is omitted.
} 
Table 4

Characteristics of Prescription Drug User Fee Act NME-Year Observations

\begin{tabular}{lccccc}
\hline Variable & Mean & SD & Median & Min & Max \\
\hline Serious ADRs & 61 & 128 & 19 & 0 & 1,433 \\
Hospitalization ADRs & 44 & 97 & 13 & 0 & 1,155 \\
Death ADRs & 10 & 26 & 2 & 0 & 279 \\
Priority review & .40 & .49 & 0 & 0 & 1 \\
Accelerated approval & .11 & .31 & 0 & 0 & 1 \\
Biological product & .12 & .32 & 0 & 0 & 1 \\
Orphan product & .14 & .34 & 0 & 0 & 1 \\
FDA review & 15 & 10 & 12 & 1 & 54 \\
log(FDA review) & 2.51 & .66 & 2.48 & -.53 & 3.99 \\
BB warning & .15 & .36 & 0 & 0 & 1 \\
BB/pregnancies & .04 & .20 & 0 & 0 & 1 \\
Percent <20 years & .12 & .20 & .03 & 0 & 1 \\
Percent $\geq 65$ years & .35 & .24 & .31 & 0 & .95 \\
Percent female & .54 & .21 & .56 & 0 & 1 \\
U.S. launch lag & 19 & 51 & 0 & 0 & 432 \\
log(U.S. launch lag) & 1.27 & 1.67 & 0 & 0 & 6.07 \\
Oral sales volume & 46,652 & 142,228 & 754 & 0 & $1,550,542$ \\
log(oral sales) & 5.49 & 5.03 & 6.63 & 0 & 14.25 \\
Injectable sales volume & 156 & 664 & 0 & 0 & 9,237 \\
log(injectable sales) & 1.48 & 2.40 & 0 & 0 & 9.13 \\
Other sales volume & 7,690 & $47,727.8$ & 0 & 0 & 651,924 \\
log(other sales) & 1.06 & 3.04 & 0 & 0 & 13.39 \\
Product age & .97 & .81 & 1 & 0 & 2 \\
\hline
\end{tabular}

Note. $N=678 . \mathrm{NME}=$ new molecular entity; $\mathrm{ADR}=$ adverse drug reaction; FDA $=$ Food and Drug Administration; $\mathrm{BB}=$ black box.

riskier throughout the initial 3 years of marketing. An interaction between the launch lag variable and age also had a statistically insignificant effect.

Since simple shift and interaction variables may not adequately capture all the changes in the post-PDUFA period, we also estimated our negative-binomial model on the PDUFA-only sample of NMEs to test its robustness. Table 4 provides some summary statistics, while Table 5 presents the coefficient estimates for the sample of PDUFA NMEs. The results in Table 5 are not much different than the estimates in Table 3 . As previously was the case, priority drugs, biological products, and drugs with shorter U.S. launch lags experience additional serious adverse events ( $p \leq .05)$. After controlling for these and other risk-related factors, we found that coefficients for none of the review time variables for linear or log specifications were statistically significant.

We then tested the interactions between FDA review time and three drug novelty variables (priority products, accelerated approvals, and biological products) to see if the effect of FDA review time differs by drug novelty. None of the interactions were statistically significantly negative, and this finding indicates that an increase in the FDA review time will not reduce serious adverse events for novel drugs. In fact, the coefficient on the interaction term between FDA review time and priority review is positive in each case and is statistically significant $(p<.05)$ for death ADRs. This finding suggests that the staff at the FDA 


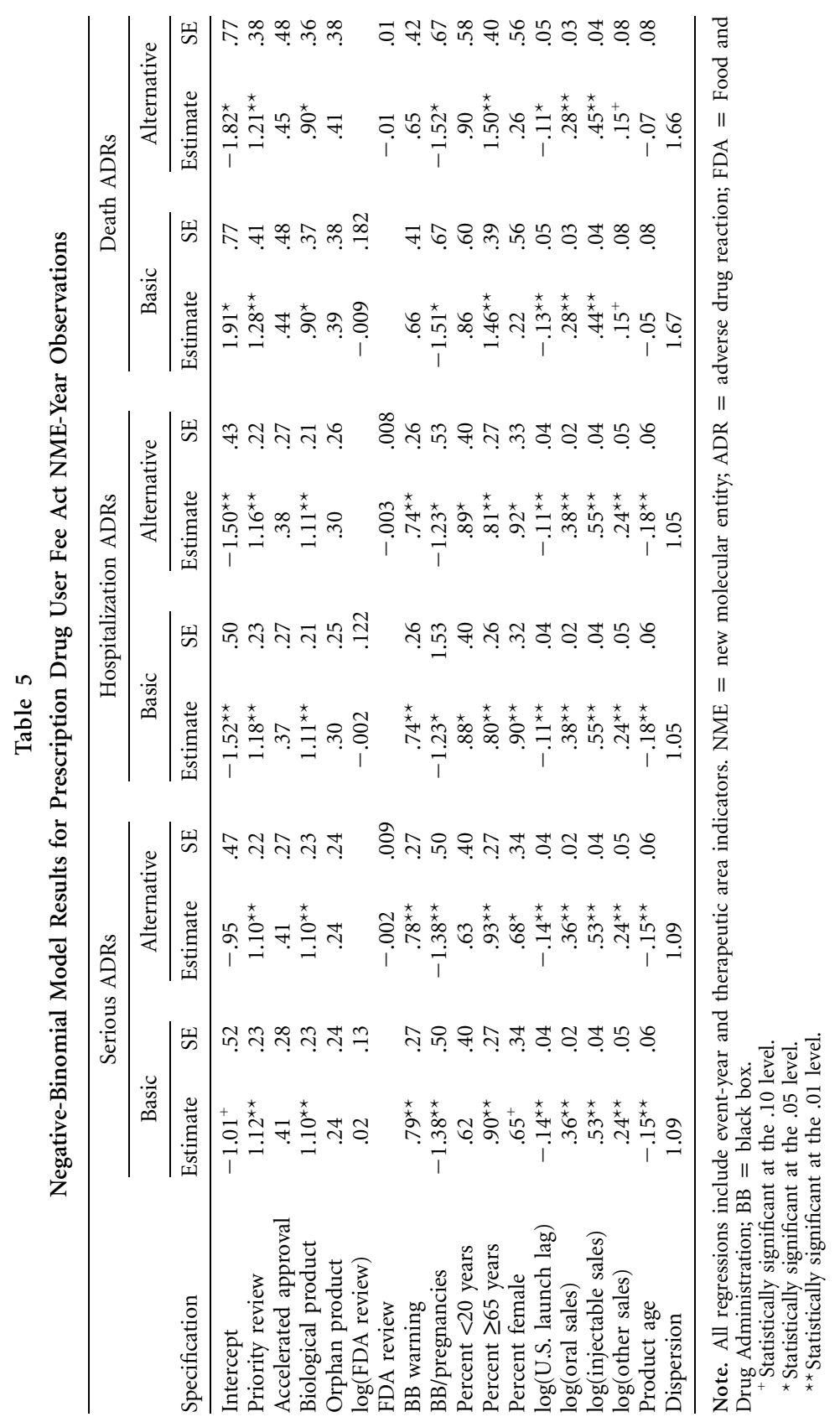


may spend more time reviewing novel but potentially dangerous drugs in the PDUFA period than it did previously. ${ }^{41}$

\subsection{The Effects of Outliers}

We next investigated the general sensitivity of our results to outliers and in particular whether this positive interaction term between novelty and review time was driven by outliers. In Figure 4, which covers the PDUFA time period, we show a plot of annual serious ADRs per 1,000 SU versus the FDA review time in months ( $n=678$; the sample is PDUFA NME-year observations). One obvious outlier is the priority-rated drug Mifiprex (generic name mifepristone), also known as RU-486, which was on the accelerated approval track, making it eligible for approval based on a surrogate end point. It is indicated for medical termination of pregnancy within the first 49 days (Table 6). This drug was subject to political controversy, and it took 54 months to gain approval after its submission in March 1996. The regimen for this drug is also one pill per prescription for each patient and therefore could have a potentially high number of ADRs per SU. These facts are consistent with its strong outlier status, shown in Figure 4 .

Table 6 presents the characteristics of the eight outliers for which annual serious ADRs per 1,000 SU are 1 standard deviation or more above the mean. ${ }^{42}$ In addition to the fact that these drugs are indicated primarily for serious, lifethreatening conditions, six of these products had black-box warnings at approval, six are priority products, and four are orphan products. The positive interaction between priority and log-transformed FDA review time was sensitive to the exclusion of the outliers shown in Table 6. It became statistically insignificant when the eight outliers were excluded. Furthermore, the coefficients for the other variables shown in Table 5 were generally unaffected by exclusion of these eight outliers. ${ }^{43}$

This analysis provides some further insights into the issue of anticipated versus unanticipated risks. In particular, the eight NMEs that were observed to have the highest ex post risk (per unit of utilization) consisted primarily of drugs for life-threatening diseases and with the presence of a black-box warning on the initial label. The presence of a black-box warning on the initial label suggests that the FDA was aware ex ante that these drugs pose much higher risks than do other NMEs, but these compounds were assessed to have important potential benefits that outweighed the risks. The issue of anticipated verses unanticipated

\footnotetext{
${ }^{41}$ In particular, the coefficients on the interaction between priority and log-transformed FDA review time were $.51(p<.10), .40(p=.14)$, and $.77(p<.05)$, respectively, for serious ADRs, hospitalization ADRs, and death ADRs. The results are similar for the full sample (Table 3 ) but have substantially smaller coefficient estimates.

${ }^{42}$ It should be noted that the first four NMEs in Table 6 (Mifeprex, Flolan, Ontak, and Mylotarg) have annual serious ADRs per 1,000 SU that are 2 standard deviations above the mean.

${ }^{43}$ The only exception was that the coefficient on the general black-box warning became insignificant $(p>.10)$.
} 


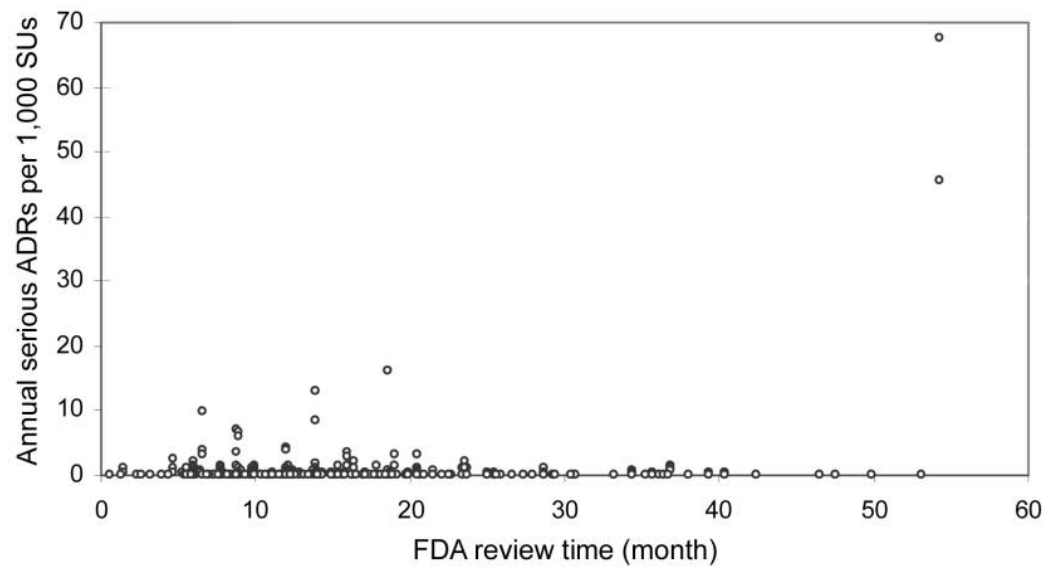

Figure 4. Food and Drug Administration review time and annual serious adverse drug reactions.

risks as reflected in the utilization and timing of black-box warnings warrants further research.

\section{Discussion}

Our findings are generally consistent with previous research, which finds no significant effect of PDUFA or shorter review times on the rate of withdrawals of new-drug approvals, either before or after the act's passage (Berndt et. al 2005; Tufts Center for the Study of Drug Development 2005; FDA 2005). However, drug withdrawals are an infrequent event. The only prior analyses of PDUFA that also employed ADRs is Olson $(2002,2004,2008)$. In contrast to our results, she finds a negative statistical relationship between ADRs and FDA review times. Compositional factors and different time periods undoubtedly play a role in explaining the differences between Olson's results and our findings. ${ }^{44}$ We also

${ }^{44}$ The most recently published paper by Olson (2008) covers the period 1990-2001 and focuses on new chemical entities (excluding most biologicals). Her study contains many of the explanatory variables used in our analysis, but there are important differences in the data sources and methodology. First, her controls for drug exposure are based on MEPS, which has prescription data beginning only in 1996. As a database, MEPS is also a weaker source of patient exposure than are industry sales audits, especially for hospital products and injectables, mental health drugs, and drugs for small patient populations. This is reflected in the smaller coefficient estimates for her utilization measures compared to our estimates. Second, her measure of foreign introductions is based on annual rather than monthly data and covers fewer countries than do IMS audits. This makes it difficult to capture the role of informational spillovers from U.S. launch lags in a precise manner. Third, her analysis of outliers is based on the absolute level of a products ADRs rather than one adjusted for a drug's exposure, as in our analysis (namely, ADRs per standard unit). Consequently, her sample of outlier observations for ADRs is distinguished by products with high consumption levels. For example, Viagra is a separate indicator variable in her regression model because it was identified as an "extreme outlier," but it is not an outlier in our analysis after taking account of patient exposure (see Table $6)$. 


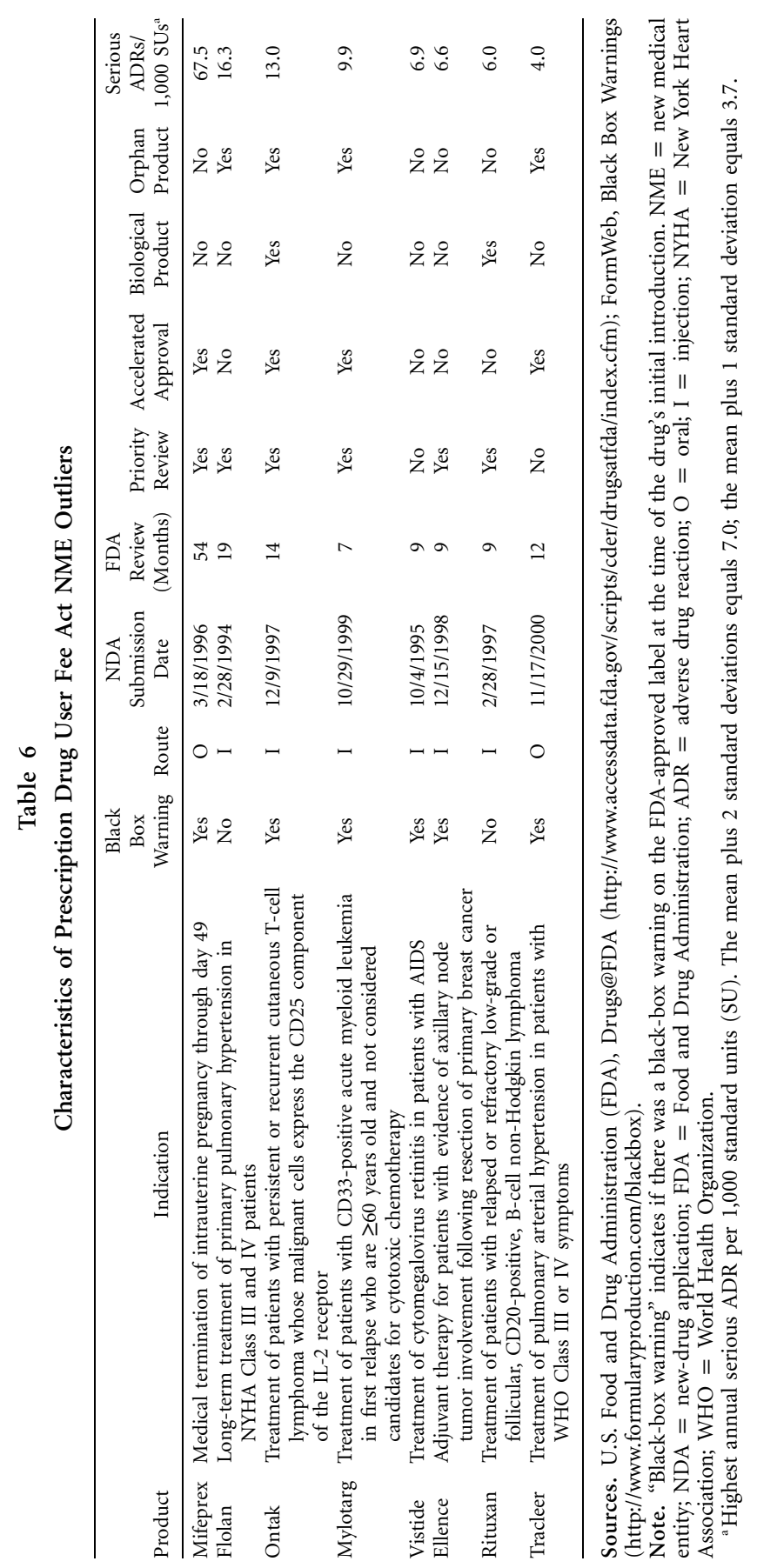


believe an important differentiating feature of our work is a richer characterization of the factors affecting new-drug risks. After adjusting for patient exposure and taking account of the higher risks associated with accelerated approval, biological entities, shorter U.S. launch lags, drugs with anticipated risks (blackbox warnings), and other factors, we find that FDA review times are not significantly related to ADRs. ${ }^{45}$ This conclusion is consistent with the countervailing effects on drug safety of PDUFA (that is, shorter review times versus expanded resources).

From a policy perspective, shorter review times mean that the FDA has less information on safety risks from foreign marketing experiences. More new drug entities, especially novel drugs, are now introduced first in the United States or simultaneously in the United States and Europe (Grabowski and Wang 2006). Our analysis indicates that a 12-month delay, other things being equal, would lead to approximately 20 fewer serious ADRs per year, compared with the case of an agent first launched in the United States. However, the opportunities for such spillover informational benefits would appear to be limited by the movement toward the harmonization of regulatory procedures across countries and with more global launches by firms. It is also important from a policy standpoint to consider the effects of regulatory delays on the availability of new drugs and incentives for drug innovation. ${ }^{46}$ Analyses of the determinants of, and returns from, pharmaceutical research and development indicate that development and review times are an important parameter affecting expenditures and returns (Grabowski, Vernon, and DiMasi 2002; Vernon et. al 2006).

Our analysis indicates that many of the important drug advances for lifethreatening diseases-those that received high priority and accelerated approval-carried black-box warnings on their initial labels. The FDA should be encouraged to continue to make these difficult benefit-risk trade-offs in a timely and objective manner and to convey this information to physicians and patients on the product's label and through other media. The net benefits from the timely availability of novel drugs appear to be substantial (Lichtenberg 2005; Olson 2004). Furthermore, Philipson et al. (forthcoming) find large consumer surplus and total surplus gains from the implementation of PDUFA.

Our results indicate that a return to the long delays of the pre-PDUFA period would have few apparent benefits and many undesirable consequences. Policy makers instead should focus on establishing a diligent postmarketing surveillance program to monitor risks and to inform physicians and patients as new knowledge becomes available. Historically, both the FDA and pharmaceutical firms

\footnotetext{
${ }^{45}$ As we discussed, the only exception is for death-related ADRs when a linear rather than a logarithmic version of the review time variable is employed (Table 3). Given the fact that review times are highly skewed, a logarithmic variable is preferred on statistical grounds. Furthermore, none of the coefficients on the review time variables, linear formulations, or logarithmic formulations are significant in the post-PDUFA period regression (Table 5).

${ }^{46}$ The effect of regulatory delays continues to be an important policy issue because research and development costs have been increasing significantly in real terms while the rate of new-drug approvals has been on a declining path in recent years (Grabowski 2004; Cockburn 2007).
} 
have devoted very modest resources for postmarketing monitoring and surveillance, compared with the expenditures for preapproval clinical trials and research and development activities (Ridley et al. 2006). This situation may be changing as a result of new legislation. The recently enacted renewal of the user-fee program, the Food and Drug Administration Amendments Act of 2007, provides dedicated user fees for new postmarket safety initiatives. Furthermore, as Mark McClellan (2007) testified before the Senate, the recent introduction of the Medicare Part D program for prescription drugs, in combination with other evolving data sources, creates opportunities for fundamental improvements in electronic infrastructure with respect to drug safety information.

\section{References}

Berndt, Ernest R., Adrian H. Gottschalk, Tomas J. Philipson, and Matthew W. Strobeck. 2005. Industry Funding of the FDA: Effects of PDUFA on Approval Times and Withdrawal Rates. Nature Reviews: Drug Discovery 4:545-54.

Cameron, A. Colin, and Pravin K. Trivedi. 2005. Microeconometrics. Cambridge: Cambridge University Press.

Cockburn, Ian. 2007. Is the Pharmaceutical Industry in a Productivity Crisis? Pp. 1-32 in Innovation Policy and the Economy, edited by Adam B. Jaffe, Josh Lerner, and Scott Stern. Cambridge, Mass.: MIT Press.

Danzon, Patricia M., and Eric L. Keuffel. 2007. Regulation of the PharmaceuticalBiotechnology Industry. Paper presented at the NBER Conference on Regulation, 2005. http://palm.nber.org/books_in_progress/econ-reg/danzon-keuffel9-14-07.pdf.

DiMasi, Joseph A. 1995. Trends in Drug Development Costs, Times, and Risks. Drug Information Journal 29:375-84.

DiMasi, Joseph A., Ronald W. Hansen, and Henry G. Grabowski. 2003. The Price of Innovation: New Estimates of Drug Development Costs. Journal of Health Economics 22:151-85.

Food and Drug Administration. 1999. Center for Drug Evaluation and Research. From Test Tube to Patient: Improving Health through Human Drugs. Special report. Washington, D.C.: US Government Printing Office.

- 2005. CDER Report to the Nation: 2004. August 22. http://www.fda.gov/cder/ reports/rtn/2004/rtn2004.htm.

Grabowski, Henry G. 2004. Are the Economics of Pharmaceutical Research and Development Changing? PharmacoEconomics 22 (Suppl. 2):15-24.

Grabowski, Henry G., John M. Vernon, and Joseph A. DiMasi. 2002. Returns on Research and Development for 1990s New Drug Introductions. PharmacoEconomics 210 (Suppl. 3):11-29.

Grabowski, Henry G., John M. Vernon, and Lacy G. Thomas. 1978. Estimating the Effects of Regulation on Innovation: An International Comparative Analysis of the Pharmaceutical Industry. Journal of Law and Economics 21:133-63.

Grabowski, Henry G., and Y. Richard Wang. 2006. The Quantity and Quality of Worldwide New Drug Introductions, 1982-2003. Health Affairs 25:452-60.

Herbst, Roy S., Dean F. Bajorin, Harry Bleiberg, Diane Blum, Desirée Hao, Bruce E. Johnson, Robert F. Ozols, George D. Demetri, Patricia A. Ganz, Mark G. Kris, Bernard Levin, Maurie Markman, Derek Raghavan, Gregory H. Reaman, Raymond Sawaya, 
Lynn M. Schuchter, John W. Sweetenham, Linda T. Vahdat, Everett E. Vokes, Roger J. Winn, and Robert J. Mayer. 2006. Clinical Cancer Advances 2005: Major Research Advances in Cancer Treatment, Prevention, and Screening-a Report from the American Society of Clinical Oncology. Journal of Clinical Oncology 24:190-205.

Institute of Medicine. 2007. The Future of Drug Safety. Washington, D.C.: National Academies Press.

Kaitin, Kenneth I., and Jeffrey S. Brown. 1995. A Drug Lag Update. Drug Information Journal 29:361-73.

Lasser, Karen E., Paul D. Allen, Steffie J. Woolhandler, David U. Himmelstein, Sidney M. Wolfe, and David H. Bor. 2002. Timing of New Black Box Warnings and Withdrawals for Prescription Medications. Journal of the American Medical Association 287:2215-20.

Lichtenberg, Frank R. 2005. Pharmaceutical Knowledge-Capital Accumulation and Longevity. Pp. 237-69 in Measuring Capital in the New Economy, edited by Carol Corrado, John Haltiwanger, and Daniel Sichel. Chicago: University of Chicago Press.

McClellan, Mark. 2007. Fundamental Improvements in Drug Safety for the TwentyFirst Century: Time for Systemic, Electronic Infrastructure. Statement of Testimony to the U.S. Senate HELP Committee. March 14. http://aei-brookings.org/admin/ authorpdfs/redirect-safely.php?fname =../pdffiles/Testimony_07-09_topost.pdf.

Olson, Mary K. 2002. Pharmaceutical Policy Changes and the Safety of New Drugs. Journal of Law and Economics 45:615-42.

- 2004. Are Novel Drugs More Risky for Patients than Less Novel Drugs? Journal of Health Economics 23:1135-58.

- 2008. The Risk We Bear: The Effects of Review Speed and User Fee Funding on New Drug Safety. Journal of Health Economics 27:175-200.

Peltzman, Sam. 1973. An Evaluation of Consumer Protection Legislation: The 1962 Drug Amendments. Journal of Political Economy 81:1949-86.

Philipson, Tomas J., Ernst R. Berndt, Adrian Gottschalk, and Matthew W. Strobeck. 2008. Cost-Benefit Analysis of the FDA: The Case of the Prescription Drug User Fee Acts. Journal of Public Economics 42:306-25.

Ridley, David B., Judith M. Kramer, Hugh H. Tilson, Henry G. Grabowski, and Kevin A. Schulman. 2006. Spending on Postapproval Drug Safety. Health Affairs 25:429-36.

Rodriguez, Evelyn M., Judy A. Staffa, and David J. Graham. 2001. The Role of Databases in Drug Postmarketing Surveillance. Pharmacoepidemiology and Drug Safety 10:407-10.

Rudholm, Niklas. 2004. Approved Times and the Safety of New Pharmaceuticals. European Journal of Health Economics 46:345-50.

Shulman, Sheila R., and Jeffrey S. Brown. 1995. The Food and Drug Administration's Early Access and Fast-Track Approval Initiatives: How Have They Worked? Food and Drug Law Journal 50:503-31.

Tufts Center for the Study of Drug Development. 2001. FDA's Fast Track Program Results in 62\% Approval Rate after First 3 Years. Tufts CSDD Impact Report, vol. 3, no. 1.

. 2005. Drug Safety Withdrawals in the US Not Linked to Speed of FDA Approval. Tufts CSDD Impact Report, vol. 7, no. 5.

Vernon, John A., Joseph H. Golec, Randall Lutter, and Clark Nardinelli. 2006. FDA New Drug Approval Times, Prescription Drug User Fees, and R\&D Spending. Working Paper no. 06-21. AEI-Brookings Joint Center for Regulatory Studies, Washington, D.C. http:// aei-brookings.org/admin/authorpdfs/redirect-safely.php?fname $=. . /$ pdffiles/phpiG.pdf.

Wardell, William, and Louis Lasagna. 1975. Regulation and Drug Development. Washington, D.C.: American Enterprise Institute. 
Wysowski, Diane K., and Lynette Swartz. 2005. Adverse Drug Event Surveillance and Drug Withdrawals in the United States, 1969-2002: The Importance of Reporting Suspected Reactions. Archives of Internal Medicine 165:1363-69. 